\title{
Generalization of Context-Specific Training in Individuals with Mild Cognitive Impairment: An Event-Related Potential Study
}

\author{
Sam C.C. Chan ${ }^{a, b}$ Tommy L.H. Lam ${ }^{a, b}$ Kenneth N.K. Fong ${ }^{a}$ \\ Marco Y.C. Pang ${ }^{a} \quad$ Chetwyn C.H. Chan ${ }^{a, b}$ \\ a Department of Rehabilitation Sciences, The Hong Kong Polytechnic University, Hong Kong, \\ China; ${ }^{b}$ Applied Cognitive Neuroscience Laboratory, The Hong Kong Polytechnic University, \\ Hong Kong, China
}

\section{Keywords}

Working memory · Mild cognitive impairment · Event-related potential · N200 · P300 .

Generalization of context-specific training

\begin{abstract}
Background: This study examined the neural processes associated with the generalization of the effect of context-specific (CS) training to noncontextual situations among individuals with mild cognitive impairment (MCI). Methods: Fourteen and 16 participants with MCI were randomly allocated to a Chinese calligraphy writing (CW) training or a control group, respectively. The CW participants learned how to write Chinese strokes in a semicursive style to construct characters, tapping on working memory functions. The control group, on the other hand, learned how to use a tablet computer without emphasis on working memory functions. They then performed two 2-back tasks with CS semicursive strokes and non-context-specific (NCS) digits. Event-related electroencephalogram signals were concurrently recorded. $\boldsymbol{R e}$ sults: The CW participants had a significantly shorter reaction time in the CS than in the NCS task $(p<0.05)$. They showed significantly longer latency in working memory updating (N200; $\left.t_{11}=4.70, p=0.05\right)$ and shorter latency in the evaluation of visual representation ( $P 300 ; t_{12}=$ $4.67 ; p=0.05$ ) than the control group when performing the 2-back CS task. Shorter P300 latency was also revealed in the 2 -back NCS task $\left(t_{12}=5.15, p=0.041\right)$, suggesting a possible generalization of the training effect among the $\mathrm{CW}$ participants. Conclusion: The results suggest that CS working memory is likely to be generalized to NCS domains among individuals with MCI. Future research should extend the scope of the generalization and apply it beyond experimental conditions.




\section{Introduction}

The decline of working memory is a common early symptom among individuals with mild cognitive impairment (MCI) [1]. Individuals with MCI were found to have a higher tendency of developing dementia later in their life $[1,2]$. Much effort has been paid to design working memory training with the aim of delaying the deterioration of individuals with MCI and to a certain extent improving their ability [3]. Behavioral studies indicated that the enhancement effects by working memory training were generalizable to contexts of stimuli different from those employed in the training [4-6]. The transferability may rely on whether one could integrate the new information in the working memory network through rehearsal and visualization [4]. More evidence is needed to show how the training effort could be transferred to new or untrained contexts.

The working memory process using an $n$-back task was found to be associated with an event-related potential (ERP) component (P200/N200) elicited over the parietal area [7, 8]. This component was found to be preceded by N160, which is related to selective attention [5, 7]. The amplitude of P200 became more positive when the working memory load was increased among healthy adults and elderly [7] as well as those with MCI [9]. N200 was reported to be associated with working memory updating among healthy [10] and older adults [7]. Individuals with progressive MCI were found to have diminishing P200/N200 when compared with those with stable MCI [11]. Similarly, 1-year follow-up of individuals with progressive MCI indicated a more prominent decrease in the amplitudes of P200/N200 $[8,9]$. Besides the amplitudes, the latency of P200/N200 showed a significant delay among individuals with progressive MCI [8]. These results suggest that P200/N200 elicited over the centroparietal area is a useful marker for reflecting working memory deficit among individuals with MCI. They also support the validity of using an $n$-back task, which involves encoding, rehearsal of and updating information for testing working memory of individuals with MCI.

Parietally distributed P300 is another ERP marker, which was found to be associated with categorizing or evaluating visual representation in the working memory buffer $[10,12]$. Less positive P300 was associated with an increased working memory load among younger subjects $[13,14]$. When compared with younger subjects, the distribution of P300 among older subjects was more centrofrontally distributed $[15,16]$. Individuals with MCI showed a latency of [17] and less positive P300 [18] than their cognitively intact counterparts. This means that individuals with MCI have a less efficient evaluation of visual representation which demands working memory.

This study aimed to investigate the neural processes associated with the generalization of the gain in working memory based on context-specific (CS) training to noncontextual situations among individuals with MCI. In particular, we are interested in understanding the role of the P200/N200 complex in reflecting working memory. The CS training employed in this study was 16 sessions of learning Chinese calligraphy writing (CW). The rate-limiting processes were decomposition and transformation of strokes of characters into different styles before writing. Specifically, the MCI participants were involved in reading Chinese characters presented in Kai script (more square shape and distinct strokes) and then writing them in Hang script (more round shape and strokes run into each other). This requires encoding, memory recall, updating and transformation of the strokes and characters across the 2 types of script before the actual writing with a brush. The contextual and noncontextual experimental tasks for eliciting the ERP components were 2-back tasks presenting Kai script strokes and digits, respectively. We hypothesized that MCI participants who received the CW training would have an enhanced working memory load and updating function as reflected by less diminished centroparietally distributed P200/N200 after the training elicited over the 
Table 1. Demographics and neuropsychological test scores of the context-specific and control groups

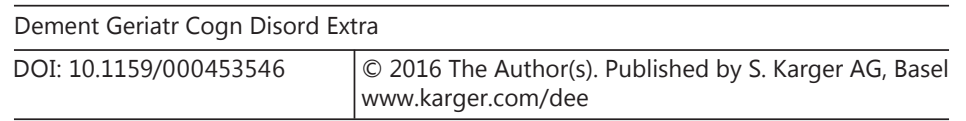

Chan et al.: Generalization of Context-Specific Training in Individuals with Mild Cognitive Impairment: An Event-Related Potential Study

\begin{tabular}{lcll}
\hline & CW group & Control group & $\begin{array}{l}\text { Signifi- } \\
\text { cance }\end{array}$ \\
\hline Subjects, $n$ & 14 & 16 & - \\
Female, $n$ (\%) & $9(64.3 \%)$ & $15(93.8 \%)$ & - \\
Age (SD), years & $65.9(5.0)$ & $66.4(3.65)$ & n.s. \\
C-MMSE score (SD) & $27.1(1.7)$ & $28.3(1.6)$ & n.s. \\
HK-MoCA (SD) & $25.5(1.7)$ & $25.4(2.1)$ & n.s. \\
AMIC & $3.5(1.2)$ & $3.1(1.1)$ & n.s. \\
DST-B & $3.4(1.5)$ & $3.4(1.5)$ & n.s. \\
Time difference between & & & \\
$\quad$ CTT part 1 and 2 & $61.6(34.6)$ & $66.3(47.2)$ & n.s. \\
Subjective rating on & & & - \\
$\quad$ calligraphy performance & $3.4(0.9)$ & - & - \\
1 & $3.6(0.8)$ & - & - \\
2 & $3.3(0.9)$ & - & - \\
3 & $3.6(0.9)$ & - & - \\
4 & $3.4(0.9)$ & - & \\
\hline
\end{tabular}

CW, Chinese calligraphy writing; C-MMSE, Chinese Mini-Mental State Evaluation; HK-MoCA, Hong Kong Montreal Cognitive Assessment; AMIC, Abbreviated Memory Inventory for Chinese; DST-B, Digit Span Test-Backward; CTT, Color Trails Test; 1, quality of learning basic strokes; 2, quantity of learning basic strokes; 3 , quality of writing basic stroke in a character; 4 , quantity of writing basic stroke in a character; n.s., not significant $(p>0.05)$.

centrofrontal regions in both the contextual and noncontextual conditions compared with those who did not receive the training. As for parietally distributed P300, it was hypothesized that the latency of P300 was reduced in the CW group in both the contextual and noncontextual conditions.

\section{Methods}

\section{Participants}

Thirty MCI participants were recruited from local community day activity centers. Fourteen and 16 participants were randomly allocated into the Chinese CW group (CS) and the computer tablet learning group (control), respectively (Table 1). The mean age of the participants in the CS group was 65.9 years $(S D=5.0)$, with the majority of them being female (64.3\%). They fulfilled the MCI criteria of a Clinical Dementia Rating Scale (CDR) score of 0.5 ("questionable dementia") [2]. The scores on the Chinese version of the Mini-Mental State Examination [19] were 27.1 (SD = 1.7) and 28.3 (SD = 1.6) and those on the Hong Kong Montreal Cognitive Assessment [20] were $25.5(\mathrm{SD}=1.7$ ) and $25.4(\mathrm{SD}=2.1$ ), respectively. The scores on the Abbreviated Memory Inventory for Chinese of the participants in the CW and control groups were $3.5(\mathrm{SD}=1.2)$ and $3.1(\mathrm{SD}=1.1)$, respectively (Table 1$)$. Comparisons of the demographic characteristics and scores on the clinical measures indicated no statistically significant between-group differences $(p>0.05)$. The study was conducted with the approval from the Departmental Research Ethics Committee, Department of Rehabilitation Sciences, the Hong Kong Polytechnic University. The objective and procedure of the study was explained to all the participants before they gave their informed consent. 
Chan et al.: Generalization of Context-Specific Training in Individuals with Mild

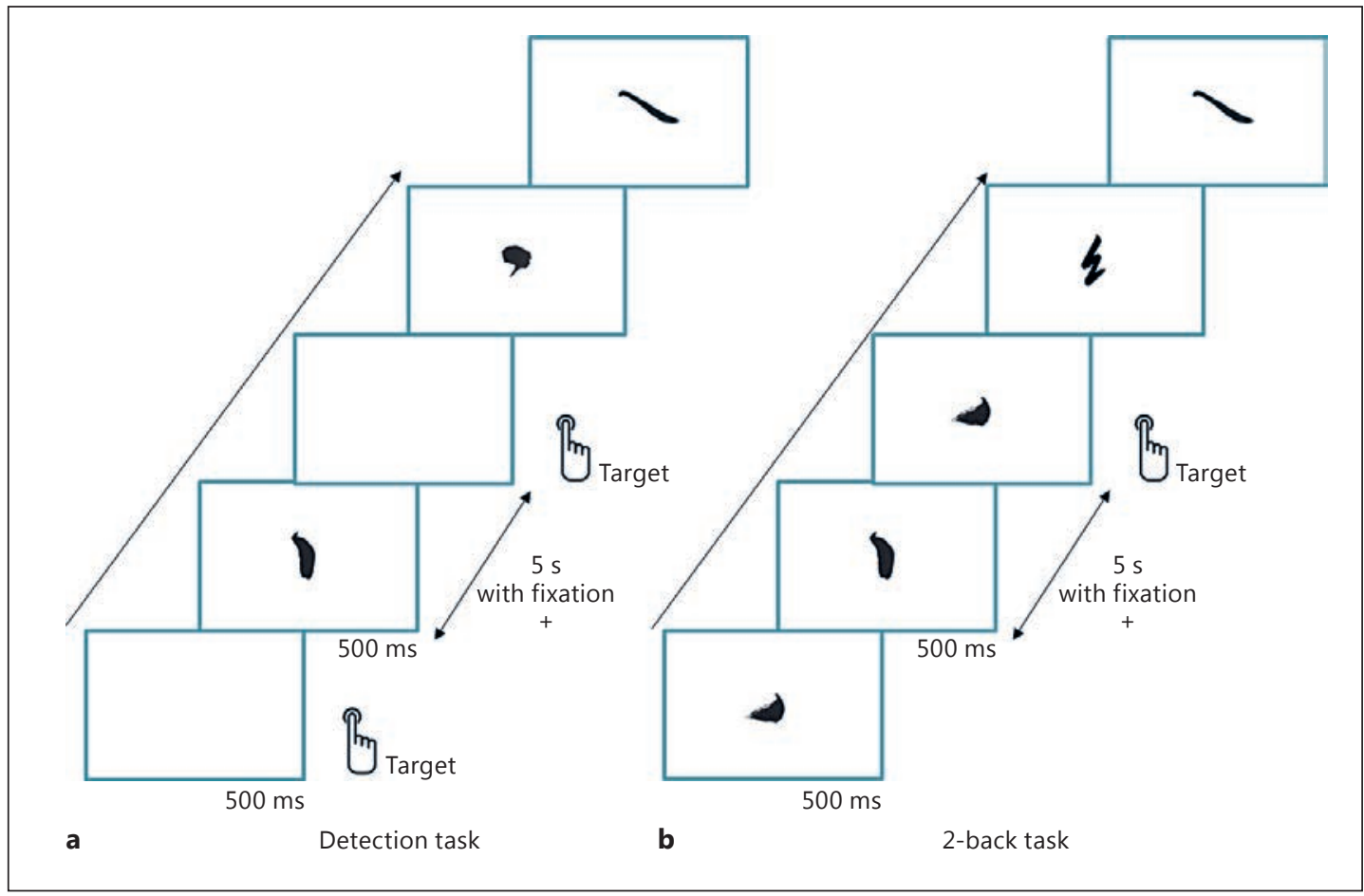

Fig. 1. Diagrammatic representation of the detection task (a) and Chinese stroke 2-back task (b).

\section{Stimuli of the 2-Back Tasks}

The two 2-back tasks for capturing cortical activities of the participants were different in terms of presenting contextual or noncontextual stimuli. Contextual stimuli were composed of strokes of the Hang script or semicursive strokes (行書; e.g. 它 in 安) and were presented in similar sizes $\left(2 \times 2.5^{\circ}\right.$ visual angle). There were altogether 20 different strokes among which 1-3 strokes were selected, composed and mounted on each stimulus (Fig. 1a, b). To ensure visuospatial processing, not all strokes presented in the stimuli carry a phonetic sound. The number of pixels of the strokes ranged from 1,275 (smallest for \) to 4,946 (largest for 幺) appearing in white on a black background. The noncontextual stimuli were 10 single-digit Arabic numbers of 0-9 that appeared in Arial font. The pixel density and size of the digits were similar to those of the semicursive stroke images.

\section{2-Back Task Design}

The 2-back task design is similar to the one described in Missonnier et al. [9]. Each participant received training on the task-taking process from a research assistant. There were 2 task conditions: contextual and noncontextual. The contextual condition presented Hang script or semicursive stroke images while the noncontextual condition presented single-digit images. All visual images, contextual or noncontextual, were presented for $500 \mathrm{~ms}$ followed by a 5,000-ms fixation cross during which the participant had to make a response (Fig. 1a, b). The participant had to press the right key on the keyboard when the image just seen was identical to the one presented 2 trials back (target). On the other hand, the participant had to press the left key when the image was not identical to the one presented 2 trials back (nontarget). The control task was to detect a single digit presented on the screen, i.e., the participant had to press the right key when viewing a blank screen or the left key when viewing a single digit. 
Chan et al.: Generalization of Context-Specific Training in Individuals with Mild

Cognitive Impairment: An Event-Related Potential Study

There were 96 trials (target:nontarget $=1: 3$ ) in each of the 2 task conditions and the control task. The trials were pseudorandomly organized into 3 blocks. The sequence of the blocks was randomized for each participant. The reaction time, accuracy and electroencephalogram (EEG) signals were recorded when the participant performed the tasks.

\section{Chinese CW (CS) Training}

The design and protocol used was part of a clinical randomized-controlled trial conducted by the authors of this paper [21]. There were 161.5 -h sessions delivered twice a week for 8 weeks. The learning of writing Chinese calligraphy was in a group (6-8 participants) led by a calligraphy master and a research assistant. During the earlier sessions, basic Chinese strokes in the Hang script or semicursive style (行書) were selected for the CW practice, such as dots ( $\backslash$ ), straight strokes ( I ), and curved strokes ( $)$ ). The participants paid attention and encoded the details of the shapes by first using a custom-designed app followed by practice with an ink brush. These basic strokes were the building blocks for whole characters. During later sessions, under the master's demonstration and feedback, the participants learned to apply the components to construct individual characters when being shown characters with a standard Kai script or angular style (楷書). This involved recalling the learned basic strokes, rehearsing them and relating visuospatial relationships while constructing the individual character. For example, $\neg \bullet)$ visuospatially formed the character 安 (meaning "peace”).

\section{Tablet Computer Training (Control)}

The format and schedule of the tablet computer training were the same as of the CW training. This was the control task used in the clinical trial study mentioned above [21]. The participants were involved in learning the functionality of an Apple iPad. The session was run by a research assistant. As a control, the participant was involved in writing Chinese characters in Hang or semicursive style on a custom-designed app in each session. The main difference from the CW training was that participants in this group were not required to rehearse semicursive style strokes and construct the semicursive style characters.

\section{Procedure for Data Collection}

Each participant was invited to attend the experiment session after having completed the $\mathrm{CW}$ training. The participants were asked to complete a demographic questionnaire and were tested with neuropsychological tests. After each participant had received training on the experiment, he or she was then seated comfortably in a distraction-free chamber and participated in the cognitive experiment while the behavioral and electrophysiological signals were recorded.

\section{Behavioral Measures for MCI}

Digit Span Test-Backward (DST-B) [22] and Color Trails Test (CTT) [23, 24] were administered to measure cognitive functions of the participants after the calligraphy training had been completed. The performance on CW was measured with a custom-made measure. The performance measure included quality and quantity of the strokes and characters assessed by the instructor who conducted the calligraphy training against a 5-point scale $(1=$ poorest and $5=$ best). The areas of assessment were: (1) quality of writing strokes, (2) quantity of writing strokes, (3) quality of writing characters, (4) quantity of writing characters, and (5) overall quality of the writing. Overall performance was calculated by averaging the scores from the 5 areas. 
Chan et al.: Generalization of Context-Specific Training in Individuals with Mild

Cognitive Impairment: An Event-Related Potential Study

\section{EEG Acquisition}

The EEG signals were captured by CURRY Scan 7 Neuroimaging Suite (NeuroScan Labs, Sterling, VA, USA) and amplified by a head box of the SynAmps2 Digital DC EEG amplifier. The 64-channel 90-mm Ag/AgCl-sintered electrode was mounted on a QuikCap (NeuroScan Labs) with the position configuration predefined according to the SynAmps2 Digital. The vertical electrooculograms were recorded with electrodes above and below the left orbit while the horizontal ones were acquired from electrodes at the external canthus of both eyes. The montage was referenced to the left and right bony mastoid processes, and the ground electrode was located on the forehead in front of the vertex electrode $(\mathrm{Cz})$. Impedances of all recording electrodes were kept at or below $5 \mathrm{k} \Omega$ throughout the whole experiment. The sampling rate of EEG signals was $1,024 \mathrm{~Hz}$. The timing of all the presented stimuli synchronized by STIM2 was recorded along with EEG signals (NeuroScan Labs).

\section{Offline EEG Preprocessing of Electrophysiological Data}

CURRY Scan 7 was used for offline preprocessing of the EEG data captured from the participants (NeuroScan Labs). The signals were first re-referenced between 2 mastoid process electrodes followed by bandpass filtering with $0.1-30 \mathrm{~Hz}$ ocular artifact reduction. EEG signals between $100 \mathrm{~ms}$ prestimulus and 1,000 ms poststimulus were epoched. After baseline correction, epochs with amplitudes larger than $100 \mu \mathrm{V}$ were rejected. Independent component analysis was used to determine the time windows for N160, P200/N200 and P300. Grand averages of latencies and amplitudes for each of the components were submitted for statistical analyses.

\section{Data Analysis}

Between-group differences in the scores of the neuropsychological measures were assessed with an independent $t$ test. Three-way repeated-measures ANOVAs Group (experimental vs. control) $\times$ Stimulus (calligraphy strokes vs. digit) $\times$ Task (2-back vs. detection) assessed the differences in the accuracy rate and reaction time on the calligraphy stroke and digit 2-back tasks. For the electrophysiological data, amplitudes and latencies of the N160, P200/N200 and P300 were obtained at the midline (Fz, Cz and Pz) and lateral (left: F3, C3 and P3 and right: F4, C4 and P4) electrodes. There were 2 models. For the midline electrodes, 4-way repeated-measures MANOVAs Group $\times$ Stimuli $\times$ Task $\times$ Midline Electrode assessed the differences in the amplitudes and latencies of each of the ERP components. A 5-way Group $\times$ Stimuli $\times$ Task $\times$ Laterality (left vs. right) $\times$ Lateral Electrode model was used for testing the differences at the lateral sites. The significant level was set at $p<0.05$ for the full model and at $p<0.01$ for all post hoc comparisons. Besides, Pearson's correlation between the significant ERP results and scores on the behavioral tests were computed. The significant level was also set at $p<0.05$.

\section{Results}

\section{Behavioral Data}

No significant between-group differences were revealed in the scores of the DST-B, CTT and CW performance test $(p>0.05)$ (Table 1). All the main effects and their interactions on the accuracy rate and reaction time on the two 2-back tasks were not statistically significant $(p>0.05)$ (Table 2). Nevertheless, post hoc comparisons indicated that the CS group had significantly shorter reaction times than the non-CS (NCS) group for both the calligraphy stroke 2-back task (792.9 ms [SD $=187.9]$ vs. $\left.1,033.3 \mathrm{~ms}[\mathrm{SD}=281.1] ; t_{28}=2.71, p=0.01\right)$ and the digit 2-back task (734.7 $\mathrm{ms}[\mathrm{SD}=185.6]$ vs. $\left.874.9 \mathrm{~ms}[\mathrm{SD}=173.2] ; t_{28}=2.14, p=0.04\right)$. 
Table 2. Accuracy and reaction time (ms) of the Chinese and digit 2-back and detection tasks of the CW $(n=14)$ and control groups $(n=16)$

\begin{tabular}{llllcl}
\hline & & \multicolumn{1}{l}{ CW } & Control & Significance \\
\hline Accuracy & \multirow{2}{*}{ Chinese } & 2-back & $0.7(0.2)$ & $0.7(0.2)$ & n.s. \\
& & detection & $0.9(0.1)$ & $0.9(0.2)$ & n.s. \\
& \multirow{2}{*}{ Digit } & 2-back & $0.8(0.1)$ & $0.8(0.1)$ & n.s. \\
& & detection & $0.9(0.1)$ & $0.9(0.1)$ & n.s. \\
Reaction time, & Chinese & 2-back & $792.9(187.8)$ & $1,033.3(281.1)$ & $\mathrm{p}=0.01$ \\
ms & & detection & $656.0(97.8)$ & $770.4(243.0)$ & n.s. \\
& \multirow{2}{*}{ Digit } & 2-back & $734.7(185.6)$ & $874.9(173.2)$ & $\mathrm{p}=0.04$ \\
& & detection & $637.1(108.5)$ & $722.4(164.2)$ & n.s. \\
\hline
\end{tabular}

CW, Chinese calligraphy writing; SD, standard deviation; n.s., not significant $(p>0.05)$. Electrophysiological data with SD in parentheses.

\section{Electrophysiological Data}

The results of the independent component analyses suggested specific time windows for capturing the ERPs: N160 (115-160 ms), P200/N200 (165-295 ms), and P300 (305-500 $\mathrm{ms}$ ). The grand average waveforms of these 3 components are shown in Figure $2 \mathrm{a}$ and $\mathrm{b}$.

\section{N160}

The Group $\times$ Stimulus $\times$ Task $\times$ Midline Electrode interaction effects on the amplitude of $\mathrm{N} 160$ were marginally significant $\left(\mathrm{F}_{2,56}=3.58, p=0.075\right)$. Among the control group, the Stimulus $\times$ Task $\left(\mathrm{F}_{1,15}=4.99, p<0.034\right)$ and the Stimulus $\times$ Midline Electrode effects were statistically significant $\left(\mathrm{F}_{2,30}=4.07, p=0.027\right)$. The amplitude at $\mathrm{Pz}$ was significantly more negative in the calligraphy stroke than in the digit 2-back task $\left(t_{15}=2.60, p=0.020\right)$.

\section{N200/P200}

The Group $\times$ Stimulus $\times$ Task effect on the latency of N200 was statistically significant $\left(\mathrm{F}_{2,26}=5.30, p=0.030\right)$. The Stimulus $\times$ Task effects were found to be only significant in the CS group $\left(F_{1,11}=11.14, p=0.01\right)$. Post hoc comparisons further indicated that the participants in the CS group showed significantly longer latency in N200 in the calligraphy stroke 2-back task than in the detection task $\left(t_{11}=4.70, p=0.05\right)$. In contrast, the participants in the CS group showed significantly shorter latency in N200 in the digit 2-back task than in the detection task $\left(t_{12}=5.94, p=0.03\right)$.

\section{P300}

The Group $\times$ Stimulus $\times$ Task effects on P300 were found to be significant $\left(\mathrm{F}_{1,27}=7.32\right.$, $p=0.012$ ). Post hoc comparisons suggested a significant Stimulus $\times$ Task effect in the CS group $\left(F_{1,12}=4.67, p=0.05\right)$. Post hoc tests further revealed that this group of participants had longer latency while performing the calligraphy stroke 2-back task than while performing the digit 2 -back task $\left(t_{12}=4.67 ; p=0.05\right)$. Besides, the same group performed the digit 2 -back task with a shorter latency than the digit detection task $\left(t_{12}=5.15, p=0.041\right)$. In contrast, only the Stimulus $\times$ Midline Electrode interaction effect was obtained in the control group $\left(\mathrm{F}_{2,30}=\right.$ $4.03, p=0.028)$. Post hoc tests revealed that the latencies of the calligraphy stroke stimuli at the midline electrodes were shorter than the latencies of the digit stimuli $\left(t_{12}=4.13-5.02\right.$, $p<0.05)$. 


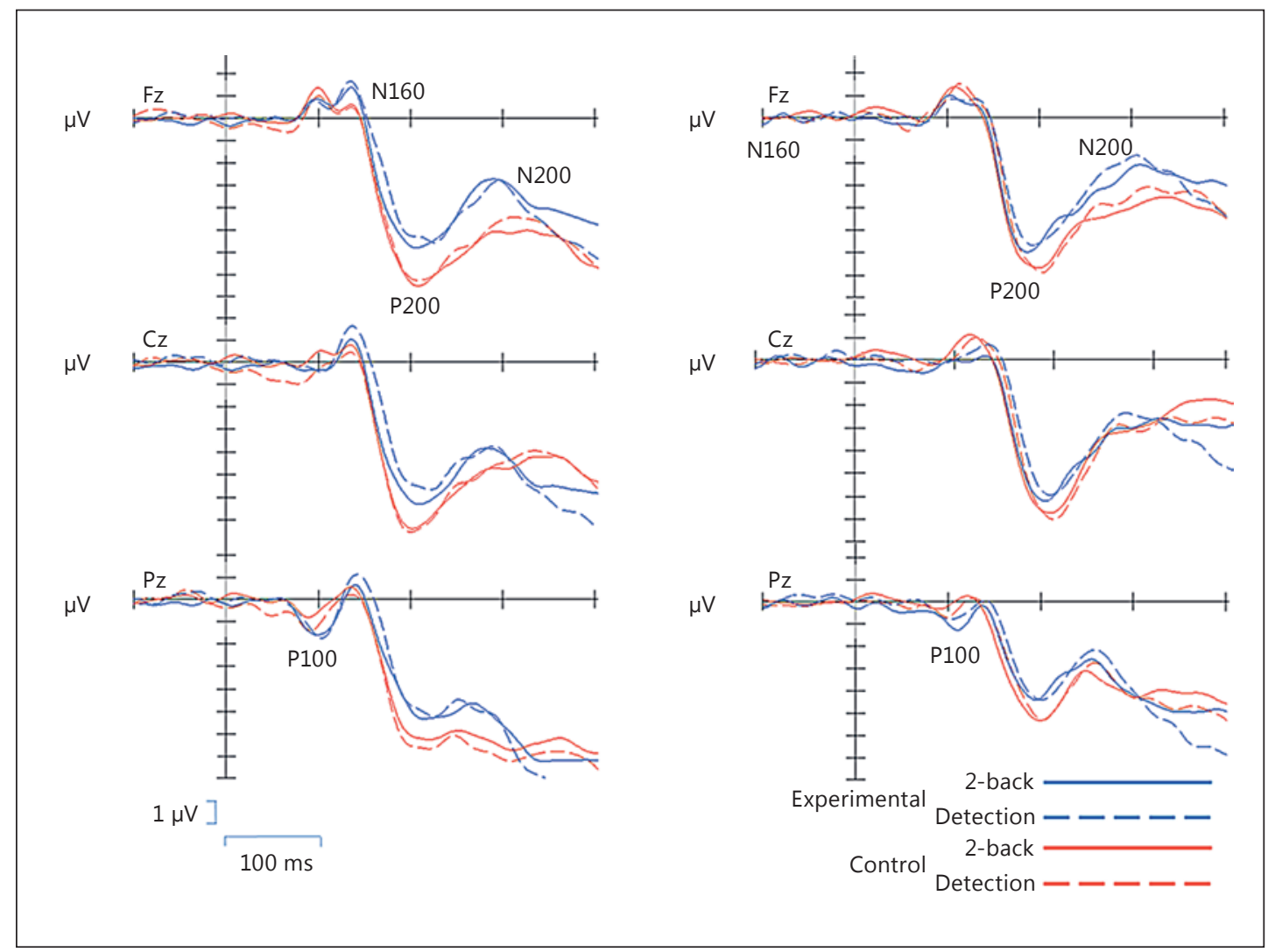

Fig. 2. Grand average waveforms at midline electrode sites (Fz, $\mathrm{Cz}$ and $\mathrm{Pz}$ ) of the Chinese stroke (a) and digit 2-back (b) tasks in the experimental (CW) and the control groups.

\section{Correlation Analysis between Behavioral and Electrophysiological Data}

The amplitudes of $\mathrm{N} 200$ at $\mathrm{Fz}$ and $\mathrm{Cz}$ in the Chinese 2-back task condition in the CS group were moderately correlated with the time required to complete the CTT part $1(0.40<r<$ $0.51, p<0.05$ ) (Table 3). The amplitudes of N200 and P300 at midline sites during both 2 -back tasks in the CS group were moderately correlated with the subscores of performance of CW $(0.54<r<0.74, p<0.05)$. No significant correlations were revealed between the amplitudes of N200 or P300 elicited at the midline or lateral sites and the scores in the DSB or CTT part 2 (e.g. completion, error and self-correction time) $(p>0.05)$.

\section{Discussion}

This study aimed to investigate the neural processes associated with the generalization of the training effects gained from CW, i.e. the visualization and transformation of visual images in working memory, to noncontextual situations among individuals with MCI. The results revealed that both $\mathrm{N} 200$ and P300 components were modulated in the CW training group. N200 was delayed across all sites during the calligraphy stroke 2-back task among the participants who received CS training. This compared favorably with those in the NCS group suggesting positive effects gained by the CS group in the 16-session training. The modulation of the N200 inspection of the visual presentation in working memory, however, was not 
Table 3. Correlations of neuropsychological tests and ERP amplitudes of N200 and P300 (at central sites) of the Chinese stroke and digit 2-back tasks in the CW group

\begin{tabular}{|c|c|c|c|c|c|c|c|c|c|c|c|c|}
\hline & \multicolumn{6}{|c|}{ Chinese stroke 2-back task } & \multicolumn{6}{|c|}{ Digit 2-back task } \\
\hline & \multicolumn{3}{|c|}{ N200 } & \multicolumn{3}{|c|}{ P300 } & \multicolumn{3}{|c|}{ N200 } & \multicolumn{3}{|l|}{ P300 } \\
\hline & $\mathrm{Fz}$ & $\mathrm{Cz}$ & $\mathrm{Pz}$ & $\mathrm{Fz}$ & $\mathrm{Cz}$ & $\mathrm{Pz}$ & $\mathrm{Fz}$ & $\mathrm{Cz}$ & $\mathrm{Pz}$ & $\mathrm{Fz}$ & $\mathrm{Cz}$ & $\mathrm{Pz}$ \\
\hline \multicolumn{13}{|c|}{ CTT (part 1) } \\
\hline Time & 0.42 & 0.43 & - & - & - & - & 0.40 & 0.51 & - & 0.44 & - & - \\
\hline \multicolumn{13}{|c|}{ Calligraphy performance } \\
\hline 1 & - & 0.57 & - & 0.61 & - & - & 0.54 & - & 0.67 & 0.55 & - & - \\
\hline 2 & 0.55 & 0.57 & 0.66 & 0.71 & - & 0.69 & - & - & - & 0.66 & - & - \\
\hline 3 & - & - & - & - & - & - & - & - & - & - & - & - \\
\hline 4 & - & - & - & 0.74 & 0.62 & 0.54 & - & - & - & 0.73 & - & - \\
\hline 5 & - & - & 0.57 & - & - & - & - & - & 0.58 & - & - & - \\
\hline
\end{tabular}

CTT, Color Trails Test; 1 , quality of learning basic strokes; 2 , quantity of learning basic strokes; 3 , quality of writing basic stroke in a character; 4 , quantity of writing basic stroke in a character; 5 , overall quality of handwriting.

reflected by the changes in the DST-B, which is a measure of working memory function. Similarly, the training effect appears to modulate the latencies of P300 among the CW training group. Different from N200, the latencies of P300 were found to be shorter in the participants who performed both the calligraphy stroke and the digit 2-back tasks than in those who performed the detection task. No differences were found between the two 2-back tasks suggesting a possible generalization of the gains in the updating process in working memory from the CW training (decomposition and transformation of strokes of characters) to the processes involved in the digit 2-back task.

No modulatory effect of CW on the P200 component, which appears to be sensitive to working memory load involved in cognitive tasks [7, 9], was revealed in the 2-back CS task. In the study by Missonnier et al. [9], the P200 amplitude was found to be diminished among those with progressive MCI while they were required to engage in a 2-back task with alphabets when compared with a 1-back or detection task. This means that practicing Chinese CW may not alter working memory capacity. It could be due to the fact that, when writing each stroke, one may only need to retrieve the visual representation of the subsequent stroke from long-term memory and maintain it on the working memory platform. In other words, this would not impose a challenge on the working memory load. On the other hand, a mediating effect of CW training was found to occur on N200. Unexpectedly, the latency of N200 was found to be longer in the CW group while performing the Chinese stroke 2-back task. Though the N200 component has been reported to be related to different functions, it has been suggested that it is associated with mental processing related to match/mismatch detection [10]. Regarding the 2-back task, it may reflect neural processing while an individual inspects the maintained visual representation in working memory buffer. In the studies by Missonnier et al. [7, 8], it was found that the amplitude of the N200 component is much reduced in those who manifested progressive MCI compared with the stable counterparts. The evidence suggests that the N200 amplitude is linked to MCI progression. What was obtained in this study was that the longer latency found in the CW group receiving CS training with the Chinese stroke 2-back task represents a more in-depth processing of the visual representation in the working memory buffer.

Another piece of evidence of the effect of CS learning of Chinese calligraphy is that the CW group performed both the Chinese stroke and the digit 2-back tasks with a much shorter 
Chan et al.: Generalization of Context-Specific Training in Individuals with Mild

Cognitive Impairment: An Event-Related Potential Study

reaction time compared to the control group despite the fact that the differences obtained may not be clinically significant (Table 2). Besides, the latency of the P300 component, which reflects the evaluative functions and updating of working memory information for the next trial, was consistently found to be shorter despite the longer latency of the N200 component in the Chinese stroke 2-back task. This resulted in a shorter reaction time while the participants performed the working memory experimental task. This shows that the CW group was more efficient in manipulating the information in the working memory buffer. The late positive component, P300, has often been considered to be a biomarker for MCI status and progression. Various studies considering other executive functions also elicited P300 (e.g., oddball design) revealing similar findings regardless of the stimulus modalities (e.g., auditory stimulus). The P300 at the parietal region was consistently found to be delayed in those with MCI $[11,25]$. On the other hand, accumulating evidence suggests that the diminishing P300 amplitude may not predict progression of MCI to Alzheimer disease. Thus, shortened latency of the P300 component revealed more efficient top-down evaluative function in the CW group after practicing CW for an extended period of time. In other words, it may counteract with the declining evaluative functions in MCI individuals. As evidence does not support that P300 alteration would predict MCI progression [17], further longitudinal studies are needed to investigate the long-term effect of Chinese CW as a means to slow down $\mathrm{MCI}$ progress.

Furthermore, the moderate correlations between both N200 and P300 components and the quality of calligraphy performance rating also suggested that calligraphy training may be associated with the extent of the details of a certain stroke in the working memory buffer, rather than the amount of image generation. This somewhat supports the findings that the processes of working memory updating (N200) and the evaluation of visual representation in the working memory buffer (P300) are modulated by ongoing practice of Chinese CW. In contrast, the much weaker, yet significant, correlations between CTT and both ERP components in question imply that the calligraphy training may not be associated with attention enhancement.

Both behavioral and electrophysiological results related to the digit 2-back task indicate the possible generalization of the effect of CS training to other NCS stimuli. The reaction time for performing the digit 2-back task was reported to be shorter in the CW group. It was found that the latencies of the N200 and P300 components were significantly shorter when performing the digit 2-back task. It appears that CW may facilitate the ability to inspect the visual representation inside the working memory buffer for Chinese strokes while writing a certain stroke (N200) and then evaluating the performance (P300). Generalization effect occurs if similar enhancement is associated with these 2 components with attempts to visually rehearse and maintain digit stimuli.

Whether learning can be facilitated through specific or generalized learning has been controversial $[6,26]$. While a collection of studies supports that perceptual learning would be contextually specific [27-29], others suggest that perceptual learning could be transferred across different types of stimuli [26, 30-32]. Such an equivocal phenomenon of perceptual learning is determined by various factors: the duration of training and schedule of the training, the relative familiarity of the two stimuli and task relevance [26]. It was shown that interleaved or less frequent training could decrease the specificity effect as it would reduce the suppressive effect of repeated exposure of the stimuli with similar characteristics [30,33]. Our calligraphy program, which was scheduled twice a week spanning 8 weeks, appeared to concur with this optimal scheduling requirement. The participants were allowed to rehearse and practice the newly learned basic strokes between sessions, where they took in new materials. This would allow the intake and retrieval of new representation in the pertaining network. Other types of stimuli, including digits, could also be practiced to heighten the generalization effect. 


\section{Conclusion}

This study only adopted a cross-sectional design with a relatively small sample and the results need to be interpreted with caution. Yet, the preliminary findings revealed possible cognitive benefits of Chinese $\mathrm{CW}$ in terms of neural processes and the generalization effect to other stimulus types. It is suggested that CW could facilitate more efficient updating (N200) and evaluating (P300) of the visual representation in the working memory buffer although the empirical findings did not reveal that the working memory capacity has been enhanced. Besides, it was shown that the beneficial effect could also be generalized to nonspecific visual stimuli (e.g. digit), especially the top-down evaluative function of mental representation in the working memory buffer (P300). In the future, it would be worthwhile conducting clinical trials with a longitudinal design to examine the long-lasting effect of Chinese CW. This would further substantiate the therapeutic effects to maintain the cognitive functions of those with MCI.

\section{Acknowledgement}

This study was supported by the Health and Health Services Research Fund (2012-2015; 09101331).

\section{Disclosure Statement}

The authors declare that the study was conducted in the absence of conflicts of interest due to sponsorship or funding arrangements.

\section{References}

1 Petersen RC, Doody R, Kurz A, Mohs RC, Morris JC, Rabins PV, et al: Current concepts in mild cognitive impairment. Arch Neurol 2001;58:1985-1992.

-2 Reisberg B, Gauthier S: Current evidence for subjective cognitive impairment (SCI) as the pre-mild cognitive impairment (MCI) stage of subsequently manifest Alzheimer's disease. Int Psychogeriatr 2008;20:1-16.

-3 Ballesteros S, Kraft E, Santana S, Tziraki C: Maintaining older brain functionality: a targeted review. Neurosci Biobehav Rev 2015;55:453-477.

4 Craik FIM, Rose NS: Memory encoding and aging: a neurocognitive perspective. Neurosci Biobehav Rev 2012;35:1729-1739.

5 Iachini I, Iavarone A, Senese VP, Ruotolo F, Ruggiero G: Visuospatial memory in healthy elderly, AD and MCI: a review. Curr Aging Sci 2009;2:43-59.

6 Lenartiwicz A, Escobedo-Quiroz, R, Cohen JD: Updating of context in working memory: an event-related potential study. Cogn Affect Behav Neurosci 2010;10:298-315.

7 Missonnier P, Gold G, Leonards U, Costa-Fazio L, Michel JP, et al: Aging and working memory: early deficits in EEG activation of posterior cortical areas. J Neural Transm 2004;111:1141-1154.

8 Missonnier P, Deiber MP, Gold G, Herrmann FR, Millet P, Michon A, et al: Working memory load-related electroencephalographic parameters can differentiate progressive from stable mild cognitive impairment. Neuroscience 2007;150:346-356.

-9 Missonnier P, Gold G, Fazio-Costa L Michel JP, Mulligan R, Michon A, et al: Early event-related potential changes during working memory activation predict rapid decline in mild cognitive impairment. J Gerontol A Biol Sci Med Sci 2005;60:660-666.

10 Daffner KR, Chong H, Sun X, Tarbi EC, Riis JL, McGinnis SM, et al: Mechanisms underlying age- and performance-related differences in working memory. J Cogn Neurosci 2011;23:1298-1314.

11 Papaliagkas VT, Kimiskidis VK, Tsolaki MN, Anogianakis G: Cognitive event-related potential: longitudinal changes in mild cognitive impairment. Clin Neurophysiol 2011;122:1322-1326.

12 Donchin E, Coles MGH: Is the P300 component a manifestation of context updating? Behav Brain Sci 1988;11: 357-374. 
Chan et al:: Generalization of Context-Specific Training in Individuals with Mild

Cognitive Impairment: An Event-Related Potential Study

13 Gevins A, Smith ME, Le J, Leong H, Bennett J, et al: High resolution evoked potential imaging of the cortical dynamics of human working memory. Electroencephalogr Clin Neurophysiol 1996;98:327-348.

$>14$ Ruchkin DS, Johnson R Jr, Grafman J, Canoune H, Ritter W: Distinctions and similarities among working memory processes: an event-related potential study. Brain Res Cogn Brain Res 1992;1:53-66.

15 Li L, Gratton C, Fabiani M, Knight RT: Age-related frontoparietal changes during the control of bottom-up and top-down attention an ERP study. Neurobiol Aging 2013;34:477-488.

16 Polich J: Meta-analysis of P300 normative aging studies. Psychophysiology 1996;33:334-353.

17 Jackson CE, Snyder PJ: Electroencephalography and event-related potentials as biomarkers of mild cognitive impairment and mild Alzheimer's disease. Alzheimers Dement 2008;4(1 suppl 1):S137-S143.

18 Olichney JM, Yang JC, Taylor J, Kutas M: Cognitive event-related potentials: biomarkers of synaptic dysfunction across the stages of Alzheimer's disease. J Alzheimers Dis 2011;26(suppl 3):215-228.

19 Chiu HFK, Kee HC, Chung WS, Kwong PK: Reliability and validity of the Cantonese version of mini-mental state examination: a preliminary study. J HK College Psychiatry 1994;4:25-28.

20 Wong A, Xiong YY, Kwan PW, Chan AY, Lam WW, Wang K, et al: The validity, reliability and clinical utility of the Hong Kong Montreal Cognitive Assessment (HK-MoCA) in patients with cerebral small vessel disease. Dement Geriatr Cogn Disord 2009;28:81-87.

21 Chan CCH, Chan SCC, Derbie AY, Hui I, Tan D, et al: Can Chinese calligraphic writing enhance cognitive performance and emotional calmness in older adults with mild cognitive impairment? An RCT study. Alzheimers Dement, in preparation.

-22 Hester RL, Kinsella GJ, Ong B: Effect of age on forward and backward span tasks. J Int Neuropsychol Soc 2004;10:475-481.

23 D’Elia LF, Satz P, Uchiyama CL, White T: Color Trails Test. Odessa, Psychological Assessment Resources, 1996.

24 Dugbartey AT, Townes BD, Mahurin RK: Equivalence of the Color Trails Test and Trail Making Test in nonnative English-speakers. Arch Clin Neuropsychol 2000;15:425-431.

25 Papaliagkas V, Kimiskidis V, Tsolaki M, Anogianakis G: Usefulness of event-related potentials in the assessment of mild cognitive impairment. BMC Neurosci 2008;9:107.

-26 Qu Z, Wang Y, Zhen Y, Hua L, Song Y, Ding Y: Brain mechanisms underlying behavioral specificity and generalization of short-term texture discrimination learning. Vision Res 2014;105:166-176.

27 Adini Y, Sagi D, Tsodyks M: Context-enabled learning in the human visual system. Nature 2002;415:790-793.

28 Teich AF, Qian N: V1 orientation plasticity is explained by broadly tuned feedforward inputs and intracortical sharpening. Visual Neurosci 2010;27:57-73.

-29 Zhaoping L, Herzog MH, Dayan P: Nonlinear ideal observation and recurrent preprocessing in perceptual learning. Network 2003;14:233-247.

30 Harris H, Gliksberg M, Sagi D: Generalized perceptual learning in the absence of sensory adaptation. Curr Biol 2012;22:1813-1817.

-31 Hussain Z, Bennett PJ, Sekuler AB: Versatile perceptual learning of textures after variable exposures. Vision Res 2012;61:89-94.

-32 Tartaglia EM, Bamert L, Mast FW, Herzog MH: Human perceptual learning by mental imagery. Curr Biol 2009;19:2081-2085.

-33 Yu C, Klein SA, Levi DM: Perceptual learning in contrast discrimination and the (minimal) role of context. J Vision 2004;4:169-182. 